\title{
To what extent has the reorganization of nuclear medicine activities during the COVID-19 pandemic fulfilled medical ethics?
}

\author{
Sandra Gonzalez ${ }^{1,2}$ - David Taïeb ${ }^{1,2,3} \cdot$ Eric Guedj $^{1,2,4} \cdot$ Pierre Le Coz ${ }^{5}$ Serge Cammilleri ${ }^{1,2,4}$
}

Received: 7 July 2020 / Accepted: 18 August 2020 / Published online: 27 August 2020

(C) Springer-Verlag GmbH Germany, part of Springer Nature 2020

Dear Sir,

The coronavirus disease 2019 (COVID-19) pandemic caused by SARS-CoV-2 has produced unprecedented damage around the globe. National responses have been varied, including preventive containment measures such as lockdowns, quarantines, and curfews. In France, people were under lockdown for 55 consecutive days and had to fill out a form to leave their house. Since May 11, 2020, a gradual deconfinement has been undertaken while maintaining proper health vigilance with respect to barrier gestures, physical distancing, personal protective equipment, and travel restrictions. This has led to suspension of routine medical care and elective surgeries across the country. In this context, respecting ethical principles of medicine is fundamental, and must guide all medical decisions.

In order to comply with European and international recommendations [1,2], relayed at the national level by the French Society of Nuclear Medicine, nuclear medicine departments had to prioritize diagnostic examinations by retaining only

This article is part of the Topical Collection on Infection and inflammation

Sandra Gonzalez

sandra.gonzalez@ap-hm.fr

1 Department of Nuclear Medicine, Assistance Publique-Hôpitaux de Marseille, La Timone University Hospital, Marseille, France

2 Service de Médecine Nucléaire, Centre hospitalo-universitaire de la Timone, Centre Européen de Recherche en Imagerie Médicale (CERIMED), Aix-Marseille Université, 264, rue Saint-Pierre, 13385 Marseille, France

3 Aix-Marseille Université INSERM, UMR1068, CRCM, Marseille, France

4 Institut Fresnel, Aix-Marseille Université, CRCM, Ecole Centrale Marseille, UMR 7249, Marseille, France

5 Espace Ethique Méditerranéen, Timone University Hospital, UMR 7268 ADéS, Aix-Marseille University-EFS-CNRS,

13385 Marseille, France those that presented an "urgent" need, i.e., those whose postponement would result in a significant loss of health, and therefore be much more harmful than the risk of possibly being exposed to SARS-CoV-2 during that particular examination. It is therefore of main importance to consider whether the applications of such recommendations were in agreement with the cardinal principles of medical ethics that include autonomy, beneficence, non-maleficence, and justice.

These four principles have been proposed by Tom Beauchamp and James Childress in the late 1970s in their book entitled Principles of Biomedical Ethics [3]. These pillars have an international scope since they respond to fundamental and universal moral expectations of every human being. Therefore, they are valid for everyone, everywhere and at all times, especially during crises. These principles can guide us during ethically challenging periods when medical decisions are no longer self-evident $[4,5]$. They provide us with tools to examine our decisions in the light of deontology and recommendations of good clinical practice.

The principle of autonomy specifies that patients should participate in all medical decisions relating to their health, which implies that they have received complete, honest, and comprehensible information. Autonomy can somewhat be considered a form of freedom. According to the principle of beneficence, physicians must intend to provide health benefits to patients, as represented by the patients themselves, which may differ from the physician or the family members' thoughts. To do so, the physicians must show solicitude, support, and understanding for their patients, considering their vulnerability, level of understanding, and expressed desires. The principle of non-maleficence, which originated from the Hippocratic oath "primum non nocere" meaning "first do no harm", calls for the avoidance of all unnecessary suffering to patients. Finally, the principle of justice advocates equal consideration and access to healthcare for all persons regardless of their particularities (e.g., age, gender, skin color), their religion, or their cultural identity. 
How were these ethical principles applied by the nuclear medicine departments complying with international recommendations during the COVID-19 crisis in France?

A change in patient management was required to fulfill the safety guidelines. This has been heavily influenced by the ethical principle of non-maleficence, as to avoid exposing patients to SARS-CoV-2. The principles of autonomy and justice were equally important, given the need to limit the number of examinations available and therefore to prioritize access to these services.

Regarding the principle of beneficence, the relationship between medical and paramedical staff with patients remained practically identical to that in the pre-crisis period. The only difference was limiting physical proximity and contact time between patients and healthcare providers to minimize risks of contamination by SARS-CoV-2.

The principle of non-maleficence is the one that has required the most adaptation in all services during this period of health crisis and it has been extended to healthcare personnel as recommended by international recommendations. Two criteria were used to determine the priority for examinations: the first concerned not causing harm to patient's health by deferring an examination, for which the risk of contamination by SARS-CoV-2 outweighed the potential benefit of the examination and the second related to the department's tools to minimize as much as possible the risk of contamination for patients and the staff by applying barrier gestures and protective measures, knowing that SARS-CoV-2 can be transmitted by asymptomatic hosts. In France, during this period, screening for SARS-CoV-2 was not routinely recommended due to lack of availability with suboptimal sensitivity.

Nevertheless, patients infected with SARS-Cov-2, whose examination was considered urgent, were welcomed in most departments with the implementation of adapted circuits (scheduling at the end of the shift and/or separate entrances/ exits/waiting areas). Finally, during the lockdown period, we noticed a very significant decrease in requests for examinations, particularly for conventional scintigraphy, which seems to indicate that prescribing physicians probably applied the principle of non-maleficence by avoiding the prescription of non-urgent examinations. It should be noted that, according to these principles, no economic consideration was taken into account during this period of crisis.

The principle of justice, in the sense of equal patient care, could be applied to all the examinations that were carried out on the basis of the defined prioritization criteria.

By contrast, the principle of autonomy has not been respected for patients whose examination was postponed following the prioritization system described above. Indeed, the medical team's decision to delay nuclear imaging investigations was not shared with patients (in order to respect the principle of justice). However, they were notified orally, and, if requested by the patient, the decision could be explained by the medical team [3]. The respect of this principle was observed for patients whose examinations were maintained, since they could benefit from clear, fair, and appropriate information regarding their examinations, and their results (when requested), unless the local practice in the department was not to communicate results directly to patients after the examination. Finally, many patients whose examinations were deemed urgent and thus maintained did not wish to take the risk of coming to the hospital and refused "freely" to undergo their examinations despite the potential medical benefit being clearly explained by the medical team.

In view of the above, it would be of major interest to share our experience at a European level during the next months in order to address three unanswered questions:

Has patient selection led to involuntary harm to the health of some patients whose examinations were maintained or conversely postponed?

Should have other criteria been taken into account to prioritize examinations in a more efficient or ethical manner?

Have we sufficiently informed and, if necessary, psychologically supported the patients whose examination postponement was a source of additional concern?

In conclusion, the clinical application of the safety guidelines in daily nuclear medicine practice during the COVID-19 crisis has ensured the respect of the cardinal ethical principles of medicine. However, the principle of autonomy could not be fully respected since the postponement of some examinations was not subject to the agreement of the patients concerned. This, however, has to be balanced with the primary responsibility of physicians, which is first do no harm.

\section{Compliance with ethical standards}

Conflict of interest The authors declare that they have no conflict of interest.

Ethical approval This article does not contain any studies with human participants performed by any of the authors.

\section{References}

1. Paez D, Gnanasegaran G, Fanti S, Bomanji J, Hacker M, Sathekge $\mathrm{M}$, et al. COVID-19 pandemic: guidance for nuclear medicine departments. Eur J Nucl Med Mol Imaging. 2020;47:1615-9. https:// doi.org/10.1007/s00259-020-04825-8.

2. Tulchinsky M, Osmany S. The American College of Nuclear Medicine Guidance on operating procedures for a nuclear medicine 
facility during COVID-19 pandemic. Clin Nucl Med. 2020. https:// doi.org/10.1097/RLU.0000000000003146.

3. Beauchamp TL, Childress JF. Principles of biomedical ethics. Oxford: Oxford University Press; 2013.

4. Le Coz P. Des repères éthiques pour les décisions médicales irréversibles. Soins. 2019.

5. Gonzalez S, Guedj E, Fanti S, Lalumera E, Le Coz P, Taieb D. Delivering PET imaging results to cancer patients: steps for handling ethical issues. Eur J Nucl Med Mol Imaging. 2018;45:2240-1. https://doi.org/10.1007/s00259-018-4124-y.

Publisher's note Springer Nature remains neutral with regard to jurisdictional claims in published maps and institutional affiliations. 